\title{
Acute myeloid leukaemia in a patient with Seckel syndrome
}

\author{
Ammar Hayani, Carlos R Suarez, Zelma Molnar, Michelle LeBeau, John Godwin
}

\begin{abstract}
We report a female patient with Seckel syndrome who developed acute myeloid leukaemia at the age of 26 years. Analysis of bone marrow chromosomes showed an abnormal clone with abnormalities involving multiple chromosomes, including monosomy 7 , trisomy 8 , trisomy 11 , and loss of the long arm of chromosome 5. After treatment with chemotherapy, the patient experienced severe toxicity with profound bone marrow aplasia and died of pneumonia two months later. We suggest that patients with Seckel syndrome may be at risk of developing myelodysplasia and acute myeloid leukaemia. They may also have poor tolerance to cytotoxic therapy.
\end{abstract}

(f Med Genet 1994;31:148-149)

Seckel syndrome is an autosomal recessive disorder characterised by severe prenatal growth retardation, mental deficiency, and distinct craniofacial abnormalities including premature synostosis, receding forehead, prominent beaked nose, and micrognathia. ${ }^{12}$ Haematological abnormalities including anaemia and bone marrow failure are seen in some patients with the syndrome. ${ }^{23}$ Leukaemia in association with Seckel syndrome has not been previously reported. We present here the clinical findings and bone marrow cytogenetics in a woman with Seckel syndrome who developed acute myeloid leukaemia. A review of published cases of Seckel syndrome associated with bone marrow failure or increased chromosome fragility is also presented.

\section{Case report}

The patient was a 26 year old female born with severe microcephaly and microsomy. At the age of 12 weeks, her weight was $1720 \mathrm{~g}$, length $49 \mathrm{~cm}$, and head circumference $28 \mathrm{~cm}$. During early childhood, she was evaluated by physicians at two outside medical institutions and was diagnosed as having Seckel syndrome. A complete blood count done at the age of 27 months was as follows: $\mathrm{Hb} 100 \mathrm{~g} / \mathrm{l}$, platelet count $210 \times 10^{9} / 1$, reticulocyte count $1 \cdot 1 \%$, and leucocyte count $4 \cdot 1 \times 10^{9} / 1$ with normal differential. A repeat complete blood count at the age of 5 years (the last reported before the present illness) showed: $\mathrm{Hb} 104 \mathrm{~g} / \mathrm{l}$ and leucocyte count $3.3 \times 10^{9} / 1$ with normal differential. She did well without any significant acute illnesses until two weeks before admission when she developed lethargy and dizziness. She presented to the hospital with cough, fever, and pallor. There was no known history of exposure to radiation or toxins, and the family history was negative for bone marrow failure or Seckel syndrome. Physical examination showed a dwarfed 26 year old female with a weight of $14.8 \mathrm{~kg}$ and a height of $113 \mathrm{~cm}$. She was severely microcephalic with a receding forehead, hypertelorism, beaked nose, low set ears, micrognathia, and downward slanting palpebral fissures. There was no hepatosplenomegaly or lymphadenopathy. The patient had mental retardation and a distinct high pitched voice.

Complete blood count showed WBC $3 \times 10^{9} / 1$, haemoglobin $16 \mathrm{~g} / 1$, platelet count $65 \times 10^{9} / 1$, blasts $4 \%$, myelocytes $5 \%$, metamyelocytes $3 \%$, bands $28 \%$, neutrophils $33 \%$, lymphocytes $24 \%$, and monocytes $3 \%$. A bone marrow aspirate was very hypocellular. Bone marrow biopsy showed $98 \%$ cellularity with extensive infiltration with blasts. A touch preparation of the marrow biopsy also showed abundance of myeloblasts with maturation and positive cytochemical reactions in the blasts to sudan black and myeloperoxidase (FrenchAmerican-British M2 morphology). Cytogenetic analysis of 21 metaphase cells from a bone marrow sample showed the presence of a normal cell line as well as a single abnormal clone with multiple abnormalities. The karyotype was as follows: $48, \mathrm{XX},+2, \operatorname{der}(5) \mathrm{t}(5 ; 17)$ (q11;q11), $-7,+8,+11,-17,+20[14] / 46, \mathrm{XX}[7]$.

A diagnosis of acute myeloid leukaemia (AML-M2) was made. After informed consent by the parents, chemotherapy was initiated with a four day continuous intravenous infusion of daunorubicin $(0.67 \mathrm{mg} / \mathrm{kg} /$ day $)$, cytosine arabinoside $(6.67 \mathrm{mg} / \mathrm{kg} /$ day), and etoposide $(3.3 \mathrm{mg} / \mathrm{kg} /$ day), in addition to 6-thio-guanine $(3.3 \mathrm{mg} / \mathrm{kg} /$ day $\times 4$ days $)$, and dexamethasone $(0.2 \mathrm{mg} / \mathrm{kg} /$ day $\times 4$ days $)$. Intrathecal cytosine arabinoside $(30 \mathrm{mg})$ was given on day 1 of therapy. In order to avoid overdosage and excessive toxicity, the doses of all cytotoxic drugs were based on body weight according to the guidelines used for infants. The patient received one course of chemotherapy after which she experienced severe herpes simplex stomatitis and a diffuse erythematous macular-papular rash. A skin rash biopsy showed lymphocytic infiltration suggestive of drug reaction. Viral, mycobacterial, and fungal cultures of the biopsy were negative. Four weeks after chemotherapy, the patient was still profoundly pancytopenic and the bone marrow was severely hypocellular. The patient remained pancytopenic and died of pneumonia two months after the initiation 
of chemotherapy. A request for necropsy was declined by the parents.

\section{Discussion}

It is estimated that one quarter of patients with Seckel syndrome have hypoplastic anaemia. ${ }^{2-4}$ Lilleyman ${ }^{5}$ reported two patients with Seckel syndrome and hypoplastic anaemia who died at 7 and 9 years of age. Both patients had a normal peripheral blood karyotype but no chromosome breakage studies were done. Peripheral blood chromosome breakage data have been reported in four patients with Seckel syndrome. ${ }^{36}$ Two of the four patients had no increase in chromosome breakage or sister chromatid exchange. ${ }^{6}$ The remaining two patients were found to have increased chromosome breakage and one of them had pancytopenia while the other one had no anaemia. ${ }^{3}$

To our knowledge, an association between Seckel syndrome and leukaemia has not been previously reported. Patients with aplastic anaemia and those with diseases associated with increased susceptibility to DNA damage such as Fanconi's anaemia, Bloom's syndrome, and ataxia telangectasia are known to have an increased incidence of haematological and lymphoid malignancies. The status of our patient's bone marrow before leukaemia is unknown but it is possible that bone marrow hypoplasia or dyserythropoiesis was present. The blood count showed mild anaemia and leucopenia during the first few years of life; however, no blood count was available after the age of 5 years. With respect to the cytogenetic abnormalities observed, monosomy 7 , trisomy 8 , trisomy 11 , and the $\operatorname{der}(5), \mathrm{t}(5 ; 17)$ are recurring abnormalities in myelodysplastic syndrome and AML. The $\operatorname{der}(5), \mathrm{t}(5 ; 17)$ results in the loss of the long arm of chromosome 5 as a consequence of an unbalanced translocation. Abnormalities of chromosomes 5 or 7 (loss or deletion of the long arm) are frequently observed in the more aggressive forms of
MDS (refractory anaemia with excess blasts and refractory anaemia with excess blasts in transformation) raising the possibility that a myelodysplastic syndrome may have preceded the development of leukaemia.

Our patient had a very poor tolerance to chemotherapy as she experienced severe stomatitis, profound bone marrow aplasia, diffuse macular-papular rash, and fatal pneumonia. Although chromosome fragility was not specifically tested in our patient, increased susceptibility to chromosome breakage, similar to that seen in patients with Fanconi's anaemia, may have been an underlying factor behind the excessive chemotherapy induced tissue damage. ${ }^{7}$

An association between Seckel syndrome and leukaemia cannot be established based on this case report; however, we would like to suggest that patients with Seckel syndrome may be at risk of developing leukaemia. Those patients may need blood count monitoring periodically and if leukaemia or bone marrow hypoplasia is suspected, bone marrow examination with cytogenetic analysis and peripheral lymphocyte chromosome fragility studies before and after the exposure to a clastrogenic agent should be done. If cytotoxic therapy is indicated, the possibility of poor tolerance and excessive toxicity should be considered.

The authors thank Lori Vega for her assistance in preparing the manuscript.

1 Seckel HPG. Bird headed dwarfs. Basel: Karger, 1960.

Majewski F, Goecke T. Studies of microcephalic primordial dwarfism. I. Approach to delineation of the Seckel syndrome. Am f Med Genet 1982;12:7-21.

3 Butler MG, Hall BD, Maclean RN, et al. Do some patients with Seckel syndrome have hematological problems and/or chromosome breakage? Am $\mathcal{F}$ Med Genet 1987;27:645-9.

4 Upjohn C. Familial dwarfism associated with microcephaly, mental retardation and anaemia. Proc $R$ Soc Med 1955; 48:334-5.

5 Lilleyman JS. Constitutional hypoplastic anemia associated with familial 'bird-headed' dwarfism (Seckel syndrome). Am $\mathcal{F}$ Pediatr Hematol Oncol 1984;6:207-9.

6 Cervenka J, Tsuchiya H, Ishiki T, et al. Seckel's dwarfism: analysis of chromosome breakage and sister chromatid exchanges. Am F Dis Child 1979;133:555-6.

7 Gluckman E, Berger R, Dutreix J. Bone marrow transplantation for Fanconi anemia. Semin Hematol 1984:21:20-6. 